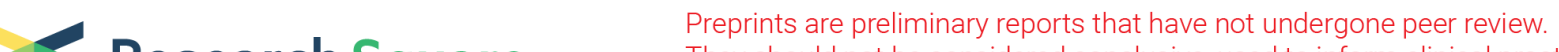 Research Square They should not be considered conclusive, used to inform clinical practice, or referenced by the media as validated information.
}

\section{A key genomic subtype associated with lymphovascular invasion in head and neck squamous cell carcinoma}

\section{Jian Zhang}

Affiliated Cancer hospital \& Institute of Guangzhou Medical University

Huali Jiang

Affiliated Donghua Hospital of Sun Yat-sen University

\section{Tao Xie}

Affiliated Cancer Hospital of Guangzhou Medical University

\section{Baiyao Wang}

Affiliated Cancer Hospital of Guangzhou Medical Unversity

\section{Xiaoting Huang}

Affiliated Cancer Hospital \& Institute of Guangzhou Medical University

Jie Lin

Affiliated Cancer Hospital \& Institute of Guangzhou Medical University

\section{Anan Xu}

Affiliated Cancer Hospital of Guangzhou Medical University

\section{Rong Li}

Affiliated Cancer Hospital \& Institute of Guangzhou Medical University

Yawei Yuan ( $\nabla$ yuanyawei@gzhmu.edu.cn )

Guangzhou Medical University Affiliated Cancer Hospital

\section{Research article}

Keywords: lymphovascular invasion, head and neck squamous cell carcinoma, hub genes, TCGA, weighted gene co-expression network analysis

Posted Date: December 6th, 2019

DOI: https://doi.org/10.21203/rs.2.18349/v1

License: (c) (1) This work is licensed under a Creative Commons Attribution 4.0 International License.

Read Full License 


\section{Abstract}

Objective: Lymphovascular invasion (LOI), a key pathological feature of head and neck squamous cell carcinoma (HNSCC), predicts poor survival. However, the associated clinical characteristics remain uncertain, and the molecular mechanisms are largely unknown.

Methods: Weighted gene co-expression network analysis was performed to construct gene co-expression networks and investigate the relationship between modules and LOI clinical trait. Functional enrichment and KEGG pathway enrichment analysis were performed for differentially expressed genesusing DAVID database. The protein-protein interaction network was constructed using Cytoscape software, and module analysis was performed using MCODE. Survival analysis and unsupervised hierarchical clustering were used to evaluate the relationships among LOI-associated genomic subtype, clinicopathological features and patient outcomes. And the potential targeted LOI molecular agents were identified with DrugBank.

Results: 10 co-expression modules in two key modules (turquoise and pink) associated with tumor LOI were identified. Functional enrichment and KEGG analysis identified turquoise and pink modules played significant roles in the progression of HNSCC. The 24 genes in two modules were identified as hub genes. Clustering analysis with seven hub genes set further divided cases into subtypes 1 and 2, which were significantly associated with pathology-determined LOI status in both cohorts. The 10-year overall survival of subtype 2 was significantly worse than that of subtype 1.

Conclusions: Our research revealed the key co-expression modules and identified seven prognostic biomarkers, including CCNA2, CNFN, DEPDC1, KIF18, KIF23, PRC1, TTK, which provide some new insights into LOI of HNSCC. Additionally, the small molecular agents may be a candidate drug for treating LOI.

\section{Background}

Head and neck squamous cell carcinoma (HNSCC), one of the most common pathological subtypes, nearly reach $90 \%$ of head and neck cancer [1]. Metastasis is the main cause of treatment failure and a important factor that affects the prognosis of HNSCC [2]. Thus, understanding the genomic changes of metastasis may be a valuable way to reduce the metastasis of lymph nodes.

In HNSCC, advanced TNM stage, histological grade and lymph node status, which indicate several high risk factors of metastatic disease, poor overall survival and disease free survival, are poor prognosis indicators $[3,4,5]$. Lymphovascular invasion (LOI) has been found to be associated with lymph node metastasis of HNSCC $[6,7,8]$. Thus, knowing effective molecular prognosticators of LOI can be a useful way to decrease the metastasis risk in HNSCC.

According to the recent studies, the clinical characteristics and parameters about LOI are still uncertain. Such as, the incidence of LOI in HNSCC was not consistent, which varied from 14 to $47 \%[9,10]$. This huge difference could be due to the small sample sizes, distribution difference and the heterogeneity of 
HNSCC. Meanwhile, it's urgent to analyze genomic and clinical characteristics of LOI based on large sample sizes clinical studies. Thus, it's critically systematic to elucidating the genomic changes and mechanism of LOI for facilitating the development of new therapeutic targets and can enhance HNSCC survival with LOI.

The Cancer Genome Atlas (TCGA) has provided comprehensive molecular features of HNSCC and offered histopathological annotations and clinical survival information of HNSCC with 10 years follow-up time. The clinical resource enabled us to systematically evaluate the relationships between LOI and gene signatures and clarify key gene modules involved in LOI of HNSCC, which provide us a comprehensive and systemic understanding of LOI not only from the genomic level but also to the prognosis level.

\section{Methods}

\section{Patient selection and data pre-processing}

Data information of HNSCC patients was downloaded from TCGA database. As shown in Table 1, RNA expression profiles and clinical survival data of 500 patients were obtained. Among these 500 patients, clinical prognosis data of 339 patients is available. According to the difference multiples $(|\log F \mathrm{C}|>1)$ and the significance threshold $(P<0.05), 2248$ genes that met the criteria were screened out as differentially expressed genes (DEG) (Additional file 1: Table S1). The intersection of DEGs from the NCBI-gene database (Additional file 2: Table S2) and OMIM database (Additional file 3: Table S3) was performed using the Venn Diagram package in $\mathrm{R}$ language. The study was approved by the Human Ethics Approval Committee of Affiliated Cancer Hospital \& Institute of Guangzhou Medical University (2019 - 290). 
Table 1

Clinical pathological characteristics of 500 patients

with HNSCC

\begin{tabular}{|lll|}
\hline Parameters & Subtype & Patients \\
\hline Age & $>61$ & 234 \\
\cline { 2 - 3 } & $\leq 61$ & 265 \\
\hline Gender & Unknow & 1 \\
\hline Lymphovascular invasion & Male & 367 \\
\cline { 2 - 3 } & Female & 133 \\
\cline { 2 - 3 } & Yes & 120 \\
\hline Pathologic stage & Unknow & 161 \\
& Stage I-II & 125 \\
\cline { 2 - 3 } & Stage III-IV & 337 \\
\hline OS times(months) & Unknow & 68 \\
\hline & $<1$ & 8 \\
\hline & $\geq 1$ & 491 \\
\hline & Unknow & 1 \\
\hline & & \\
\hline & & 219 \\
\hline
\end{tabular}

\section{Construction of co-expression module network}

The module-trait associations were considered as important clinical characteristics between the clinical phenotype and module eigengenes (MEs). As the method used by previous studies [11, 12], we analyzed the module-trait correlation and clarified the relevant modules, which were closely associated with the LOI clinical traits. For the key gene modules, gene significance (GS) and module membership (MM) mean a positive correlation level between RNA expression profiles and LOI clinical phenotype, and between RNA expression profiles and clinical MEs.

\section{Enrichment of key co-expression modules analysis}

As the method used by previous studies $[12,13]$, the aberrantly expressed mRNAs in the key gene modules were selected to perform GO function analysis and KEGG pathway analysis. For GO analysis, 
the corresponding genes were divided into by biological process (BPs) analysis. Using the KEGG analysis, genes of the key co-expression modules were used to detect the gene modules functions, and $\mathrm{P}<0.05$ was considered as statistically significant.

\section{PPI network analysis and identification of hub genes}

As method used by previous studies [14, 15], the key gene co-expression module was further explored to predict gene function correlation using STRING database with a confidence score $>0.9$. Cytoscape was employed to screen significant gene pairs in the PPI network [16]. We further screen the modules of the PPI network by molecular complex detection (MCODE) analysis. The criteria of MCODE were as follows: degree cutoff $=2$, node cutoff $=0.2$, maximum depth $=100$ and k-core $=2$. At last, the 24 genes were selected as hub genes.

\section{Survival analysis of Hub genes}

24 hub genes were analyzed using univariate survival analysis. Seven genes with significant prognostic differences were selected as characteristic genes by $P<0.05$. According to the expression profiles of characteristic genes, unsupervised hierarchical clustering was used to classify clinical samples, and Kaplan Meier was further used to explore the prognostic differences of the classified samples.

\section{Identification of small molecular drugs}

DrugBank is a comprehensive and systematic resource to explore detailed drug-target interaction information [17]. The turquoise and pink modules in PPI network were mapped onto the DrugBank database. The |connectivity scorel $>2$ was used as cutoff value to identify molecular drugs targeted with HNSCC.

\section{Statistical Analysis}

Univariate analysis was performed using SPSS 17.0 (SPSS Inc., Chicago, IL, USA). Cumulative survival time was calculated and analyzed by the Kaplan-Meier and log-rank test. The difference between two groups was tested by a chi-square test or Fisher's exact test. P-value $<0.05$ was considered statistically significant.

\section{Results}

\section{Weighted co-expression network construction and key module analysis}


We first performed the initial quality assessment using the average linkage method. Two outlier samples were removed after clustering. The remaining 339 cancer samples and 44 control samples with clinical information of LOI were used in subsequent analysis. The 2,601 variant genes in samples were with the largest variance via average linkage hierarchical clustering. To estabilish a scale-free network, the scale index (Fig. 1a) and mean connectivity (Fig. 1b, c) were calculated. We found that the power value of $\beta=7$ (scale free $R^{2}=0.85$ ) was optimum to perform further analysis (Fig. 1d). Moreover, genes with similar expression patterns could be placed into different modules via average linkage clustering. Finally, a total of 10 modules were identified (Fig. 2). The correlations between MEs and LOI trait were explored. As shown in Fig. 3a, the result indicated that 10 co-expression modules were correlated with LOI phenotypes. Figure 3B showed 10 co-expression modules was associated with cancer status, especially turquoise and pink key modules. Then, scatter diagrams of GS for MM vs. LOI status in the turquoise and pink modules were plotted, respectively (Fig. 3c, d), which showed the genes in two modules were significantly related with LOI status. The correlation values were 0.4 (turquoise) and 0.59 (pink), P-values were $1.4 \times 10^{-30}$ (turquoise) and $1.8 \times 10^{-8}$ (pink), which indicated the turquoise and pink modules were high correlations with LOI status.

\section{Enrichment analysis of key co-expression modules}

To know the function of genes in the key co-expression modules, GO and KEGG analysis were performed. GO analysis showed that the turquoise module was associated with DNA replication, mitotic nuclear division, nuclear division and DNA - dependent DNA replication. KEGG analysis found that the turquoise module was associated with cell cycle, DNA replication, mismatch repair and p53 signaling pathway (Fig. 4a, b). Similarly, GO analysis indicated that the pink module was involved in peptidase activity, negative regulation of proteolysis, negative regulation of peptidase activity and negative regulation of endopeptidase activity (Fig. 4c). These results indicated that the turquoise module and pink modules played an important role in LOI of HNSCC.

\section{PPI analysis and hub genes}

To know the hub genes in the key modules, PPI analysis of STRING database were analyzed. 89 genes in turquoise module and 38 genes in pink module were screened as candidate hub genes (Fig. 5 and Additional file 4: Table S4 and Additional file 5: Table S5). In addition, connect threshold ( $\$ 6)$ was further used to define the hub genes and then 24 genes (18 genes in turquoise module and 6 genes in pink module) were defined as hub genes. After excluding the samples with no survival information/survival time less than 1 month, 339 cancer samples were used to evaluate the prognosis of 24 hub genes. The univariate survival analysis of each hub gene was analyzed by using R-package survival. Seven genes (CCNA2, CNFN, DEPDC1, KIF18, KIF23, PRC1 and TTK1) with significant prognostic differences were screened out as characteristic genes by screening $\mathrm{P}<0.05$ (Fig. 6). 
To further validate these results, hierarchical clustering was conducted on the TCGA cohort using these seven genes, the heatmap showed patients of HNSCC were clustered into two groups: cluster $1(n=82)$ and cluster $2(\mathrm{n}=248)(\mathrm{Fig} .7 \mathrm{a})$. Compared with cluster 2, Cluster 1 had better survival probability (Fig. $7 b$ ). These results indicated that the seven genes can differentiate different LOI subtypes and seven genes maybe serve as the biomarker for LOI of HNSCC.

\section{Identification of small molecular agents}

To know the small molecular agents targeted LOI, we searched the DrugBank database and found that five drug-module interactions in turquoise module (XL844, AT7519, AT9283, Alvocidib and Nelarabine) and three drug-module interactions in pink module (Benzamidine, L-Glutamine and Zinc) may be used to target LOI (Table 2). These results indicated that XL844, AT7519, AT9283, Alvocidib, Nelarabine, Benzamidine, L-Glutamine and Zinc may provide us new approach to block metastasis of lymph nodes.

Table 2

Enriched significant of small molecules

\begin{tabular}{|llll|}
\hline module & drug & connection & P value \\
\hline pink & Benzamidine & 2 & $8.29 \mathrm{E}-07$ \\
pink & L-Glutamine & 2 & $1.28 \mathrm{E}-05$ \\
\hline pink & Zinc & 2 & 0.001325331 \\
\hline turquoise & XL844 & 2 & 0 \\
\hline turquoise & AT7519 & 2 & 0 \\
\hline turquoise & AT9283 & 2 & 0 \\
\hline turquoise & Alvocidib & 5 & $3.55 \mathrm{E}-06$ \\
\hline turquoise & Nelarabine & 2 & 0.000198791 \\
\hline
\end{tabular}

\section{Discussion}

Metastasis is the main cause of HNSCC treatment failure [18]. Nodal metastatic disease is considered as a independent factor for poor survival in $\operatorname{HNSCC}[19,20,21]$. Several clinicopathologic parameters have validated as associated with nodal metastasis, such as tumor size [9], tumor depth [22], tumor differentiation [23], histological grade [24] and LOI [4]. In this study, we performed a systematic analysis of LOI in HNSCC from the molecular level to the clinical level by comprehensive integrating genomic analysis. We found a key genomic subtype associated with lymphovascular invasion in HNSCC and small molecular agents including XL844, AT7519, AT9283, Alvocidib, Nelarabine, Benzamidine, LGlutamine and Zinc may provide us new approach to block LOI. 
With the application of sequencing techniques, genomic studies have transformed from individual genes aberrant expression to systematically integrating genomics mutation and chromatin remodel study. However, the molecular mechanisms of LOI are still unclear. TCGA database has listed several genomic landscape study of HNSCC from worldwide, which provides us a chance to integrate genomics data and understand the molecular changes of LOI. In this study, we conducted a co-expression network module of HNSCC and found the turquoise and pink modules were significantly associated with LOI. Function enrichment analysis indicated that the key gene modules function was mainly involved in the BPs of DNA replication, mitotic nuclear division, nuclear division and DNA - dependent DNA replication. Pathway enrichment analysis validated that the genes in the key module were enriched in cell cycle, DNA replication, mismatch repair and p53 signaling pathway, which indicated that the key gene modules play an important role in LOI in HNSCC.

It is essential to early diagnosis of LOI, as HNSCC patients with LOI may require more timely treatment $[25,26]$. Despite the development and application of MRI and PET-CT to assess LOI in HNSCC, the detection rate of early-stage LOI is still rarely low [27]. In this study, the hub genes in the key modules related to LOI were screened and hierarchical clustering showed that subtypes including seven genes (CCNA2, CNFN, DEPDC1, KIF18, KIF23, PRC1 and TTK1) could differentiate the risk of LOI. And the subtypes of seven genes would be potential biomarkers of LOI.

However, the targeted treatment for LOI is lacking and unreliable. DrugBank provides comprehensive molecular information about drugs and their targets for the treatment of LOI. Based on interactions of drug and key modules, we found 8 small molecular agents that could target LOI, including Benzamidine, L-Glutamine, Zinc, XL844, AT7519, AT9283, Alvocidib and Nelarabine. A recent study found that AT7519 and Alvocidib, a cyclin-dependent kinase inhibitor, has been shown to have potential for anticancer effects for cancer treatment $[28,29,30,31,32]$. XL844, a specific inhibitor of Chk1 and Chk2 kinase, has been found to effectively sensitize cancer cells to induce cell cycle arrest [33]. These results indicated the 8 small molecular agents may target hub genes of hub genes and can treat LOI of HNSCC.

\section{Conclusions}

In summary, the current study was intended to identify LOI with comprehensive bioinformatics analysis and we found a key subtype including CCNA2, CNFN, DEPDC1, KIF18, KIF23, PRC1 and TTK1 may be potential biomarkers and predict progression in LOI of HNSCC. Benzamidine, L-Glutamine, Zinc, XL844, AT7519, AT9283, Alvocidib and Nelarabine may be a candidate drug for treating LOI.

\section{Abbreviations}

LOI: Lymphovascular invasion (LOI), HNSCC: head and neck squamous cell carcinoma, DEGs: differentially expressed genes, MEs: module eigengenes, GS: gene significance, MM: module membership, WGCNA: Weighted gene co-expression network analysis, MCODE: Molecular Complex 
Detection, MRI: magnetic resonance imaging, PET-CT: positron emission tomography-computed tomography.

\section{Declarations}

\section{Ethics approval and consent to participate}

Written Informed consent was obtained from all patients before treatment and this study was approved by the institutional research ethics committee of Affiliated Cancer Hospital \& Institute of Guangzhou Medical University (2019-290).

\section{Consent for publication}

Not applicable.

\section{Availability of data and materials}

All of the data of this study has been downloaded from TCGA database (https://cancergenome.nih.gov/), OMIM database (https://www.omim.org/), NCBI gene databse (https://www.ncbi.nlm.nih.gov/gene/) and DrugBank database (https://www.drugbank.ca/).

\section{Competing interest}

The authors declare that they have no competing interests.

\section{Funding}

This work was supported by grants from the Social Science and Technology Development Key Project of Dongguan (201750715046462); Guangzhou Key Medical Discipline Construction Project Fund (B195002004042); Open Funds of State Key Laboratory of Oncology in South China (KY013711).

\section{Acknowledgements}

We would like to thank the native English-speaking scientists of Elixigen Company (Huntington Beach, California) for editing our manuscript.

\section{Author contributions}

JZ, HLJ and TX designed the research. JZ, RL, BYW, JL, AAX and XTH acquired and analyzed the data. JZ and $Y Y$ wrote the manuscript.

\section{References}

1. Torre LA, Bray F, Siegel RL, Ferlay J, Lortet-Tieulent J, Jemal A. Global cancer statistics, 2012. CA Cancer J Clin. 2015;65(2):87-108. doi: 10.3322/caac.21262. 
2. Warnakulasuriya S. Global epidemiology of oral and oropharyngeal cancer. Oral Oncol. 2009;45(45):309-16. doi: 10.1016/j.oraloncology.2008.06.002.

3. Vasan K, Low TH, Gupta R, Ashford B, Asher R, Gao K, Ch'ng S, Palme CE, Clark JR. Lymph node ratio as a prognostic factor in metastatic cutaneous head and neck squamous cell carcinoma. Head Neck. 2018;40(5):993-999. doi: 10.1002/hed.25066.

4. Wreesmann VB, Katabi N, Palmer FL, Montero PH, Migliacci JC, Gönen M, Carlson D, Ganly I, Shah JP, Ghossein R, et al. Influence of extracapsular nodal spread extent on prognosis of oral squamous cell carcinoma. Head Neck. 2016;38 Suppl 1:E1192-9. doi: 10.1002/hed.24190.

5. Liu SA, Wang CC, Jiang RS, Lee FY, Lin WJ, Lin JC. Pathological features and their prognostic impacts on oral cavity cancer patients among different subsites - A singe institute's experience in Taiwan. Sci Rep. 2017;7(1):7451. doi: 10.1038/s41598-017-08022-w.

6. Moore BA, Weber RS, Prieto V, El-Naggar A, Holsinger FC, Zhou X, Lee JJ, Lippman S, Clayman GL. Lymph node metastases from cutaneous squamous cell carcinoma of the head and neck. Laryngoscope. 2005;115(9):1561-7.

7. Yilmaz T, Hosal AS, Gedikoglu G, Onerci M, Gürsel B. Prognostic significance of vascular and perineural invasion in cancer of the larynx. Am J Otolaryngol. 1998;19(2):83-8.

8. Karahatay S, Thomas K, Koybasi S, Senkal CE, Elojeimy S, Liu X, Bielawski J, Day TA, Gillespie MB, Sinha $D$, et al. Clinical relevance of ceramide metabolism in the pathogenesis of human head and neck squamous cell carcinoma (HNSCC): attenuation of C(18)-ceramide in HNSCC tumors correlates with lymphovascular invasion and nodal metastasis. Cancer Lett. 2007;256(1):101-11.

9. Kurokawa H, Yamashita Y, Takeda S, Zhang M, Fukuyama H, Takahashi T. Risk factors for late cervical lymph node metastases in patients with stage I or II carcinoma of the tongue. Head Neck.

2002;24(8):731-6.

10. Hahn SS, Spaulding CA, Kim JA, Constable WC. The prognostic significance of lymph node involvement in pyriform sinus and supraglottic cancers. Int J Radiat Oncol Biol Phys. 1987;13(8):1143-7. 11. Lu JM, Chen YC, Ao ZX, Shen J, Zeng CP, Lin X, Peng LP, Zhou R, Wang XF, Peng C, et al. System network analysis of genomics and transcriptomics data identified type 1 diabetes-associated pathway and genes. Genes Immu. 2019;20(6):500-508. doi: 10.1038/s41435-018-0045-9.

12. Yuan L, Chen L, Qian K, Qian G, Wu CL, Wang X, Xiao Y. Co-expression network analysis identified six hub genes in association with progression and prognosis in human clear cell renal cell carcinoma (ccRCC). Genom Data. 2017;14:132-140. doi: 10.1016/j.gdata.2017.10.006.

13. Zhang Y, Wang J, Ji LJ, Li L, Wei M, Zhen S, Wen CC. Identification of Key Gene Modules of Neuropathic Pain by Co-Expression Analysis. J Cell Biochem 2017;118(12):4436-4443. doi:

10.1002/jcb.26098.

14. von Mering C, Huynen M, Jaeggi D, Schmidt S, Bork P, Snel B. STRING: a database of predicted functional associations between proteins. Nucleic Acids Res. 2003;31(1):258-61.

15. Xia WX, Yu Q, Li GH, Liu YW, Xiao FH, Yang LQ, Rahman ZU, Wang HT, Kong QP. Identification of four hub genes associated with adrenocortical carcinoma progression by WGCNA. Peer J. 2019;7:e6555. doi: 10.7717/peerj.6555.

16. Shannon P, Markiel A, Ozier O, Baliga NS, Wang JT, Ramage D, Amin N, Schwikowski B, Ideker T. 
Cytoscape: a software environment for integrated models of biomolecular interaction networks. Genome Res. 2003;13(11):2498-504.

17. Wishart DS, Feunang YD, Guo AC, Lo EJ, Marcu A, Grant JR, Sajed T, Johnson D, Li C, Sayeeda Z, Assempour N, et al. DrugBank 5.0: a major update to the DrugBank database for 2018. Nucleic Acids Res. 2018;46(D1):D1074-D1082. doi: 10.1093/nar/gkx1037.

18. Leeman JE, Li JG, Pei X, Venigalla P, Zumsteg ZS, Katsoulakis E, Lupovitch E, McBride SM, Tsai CJ, Boyle JO, et al. Patterns of Treatment Failure and Postrecurrence Outcomes Among Patients With Locally Advanced Head and Neck Squamous Cell Carcinoma After Chemoradiotherapy Using Modern Radiation Techniques. JAMA Oncol. 2017; 3(11):1487-1494. doi: 10.1001/jamaoncol.2017.0973.

19. Layland MK, Sessions DG, Lenox J. The influence of lymph node metastasis in the treatment of squamous cell carcinoma of the oral cavity, oropharynx, larynx, and hypopharynx: N0 versus N+. Laryngoscope. 2005;115(4):629-39.

20. Sessions DG, Spector GJ, Lenox J, Parriott S, Haughey B, Chao C, Marks J, Perez C. Analysis of treatment results for floor-of-mouth cancer. Laryngoscope. 2000;110(10 Pt 1):1764-72.

21. Sessions DG, Lenox J, Spector GJ, Chao C, Chaudry OA. Analysis of treatment results for base of tongue cancer. Laryngoscope. 2003; 113(7):1252-61.

22. Pentenero M, Gandolfo S, Carrozzo M. Importance of tumor thickness and depth of invasion in nodal involvement and prognosis of oral squamous cell carcinoma: a review of the literature. Head Neck. 2005;27(12):1080-91.

23. Byers RM, El-Naggar AK, Lee YY, Rao B, Fornage B, Terry NH, Sample D, Hankins P, Smith TL, Wolf PJ. Can we detect or predict the presence of occult nodal metastases in patients with squamous carcinoma of the oral tongue? Head Neck. 1998;20(2):138-44.

24. Umeda M, Yokoo S, Take Y, Omori A, Nakanishi K, Shimada K. Lymph node metastasis in squamous cell carcinoma of the oral cavity: correlation between histologic features and the prevalence of metastasis. Head Neck. 1992;14(4):263-72.

25. Solares CA, Mason E, Panizza BJ. Surgical Management of Perineural Spread of Head and Neck Cancers. J Neurol Surg B Skull base. 2016;77(2):140-9. doi: 10.1055/s-0036-1579751.

26. Bur AM, Lin A, Weinstein GS. Adjuvant radiotherapy for early head and neck squamous cell carcinoma with perineural invasion: A systematic review. Head Neck. 2016;38 Suppl 1:E2350-7. doi:

10.1002/hed.24295.

27. Lee H, Lazor JW, Assadsangabi R, Shah J. An Imager's Guide to Perineural Tumor Spread in Head and Neck Cancers: Radiologic Footprints on (18)F-FDG PET, with CT and MRI Correlates. J Nucl Med.

2019;60(3):304-311. doi: 10.2967/jnumed.118.214312.

28. Dolman ME, Poon E, Ebus ME, den Hartog IJ, van Noesel CJ, Jamin Y, Hallsworth A, Robinson SP, Petrie K, Sparidans RW, et al. Cyclin-Dependent Kinase Inhibitor AT7519 as a Potential Drug for MYCNDependent Neuroblastoma. Clin Cancer Res. 2015;21(22):5100-9. doi: 10.1158/1078-0432.CCR-15-0313. 29. Kang MA, Kim W, Jo HR, Shin YJ, Kim MH, Jeong JH. Anticancer and radiosensitizing effects of the cyclin-dependent kinase inhibitors, AT7519 and SNS032, on cervical cancer. Int J Oncol. 2018;53(2):703712. doi: $10.3892 /$ ijo.2018.4424.

30. Kong Y, Sheng X, Wu X, Yan J, Ma M, Yu J, Si L, Chi Z, Cui C, Dai J, et al. Frequent Genetic Aberrations 
in the CDK4 Pathway in Acral Melanoma Indicate the Potential for CDK4/6 Inhibitors in Targeted Therapy. Clin Cancer Res. 2017;23(22):6946-6957. doi: 10.1158/1078-0432.CCR-17-0070.

31. Hafner M, Mills CE, Subramanian K, Chen C, Chung M, Boswell SA, Everley RA, Liu C, Walmsley CS, Juric D, et al. Multiomics Profiling Establishes the Polypharmacology of FDA-Approved CDK4/6 Inhibitors and the Potential for Differential Clinical Activity. Cell Chem Biol. 2019;26(8):1067-1080. doi:

10.1016/j.chembiol.2019.05.005.

32. Roskoski R, Jr. Cyclin-dependent protein serine/threonine kinase inhibitors as anticancer drugs.

Pharmacol Res. 2019;139:471-488. doi: 10.1016/j.phrs.2018.11.035.

33. Matthews DJ, Yakes FM, Chen J, Tadano M, Bornheim L, Clary DO, Tai A, Wagner JM, Miller N, Kim YD, et al. Pharmacological abrogation of S-phase checkpoint enhances the anti-tumor activity of gemcitabine in vivo. Cell cycle. 2007; 6(1):104-10.

\section{Additional Files}

Additional file 1: Table S1 The differentially expressed genes in TCGA database.

Additional file 2: Table S2 The genes related to HNSCC in NCBI gene database.

Additional file 3: Table S3 The genes related to HNSCC in OMIM database.

Additional file 4: Table S4 The candidate hub genes in turquoise module.

Additional file 5: Table S5 The candidate hub genes in pink module.

\section{Figures}


A

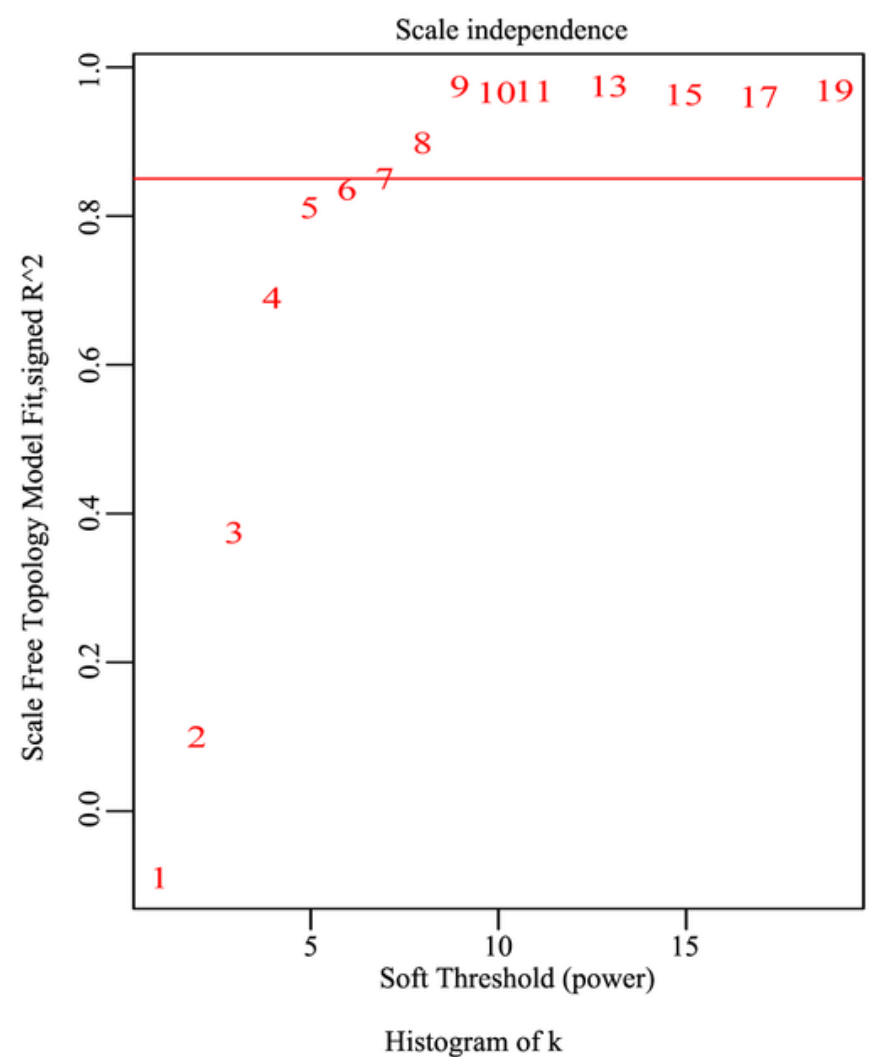

C

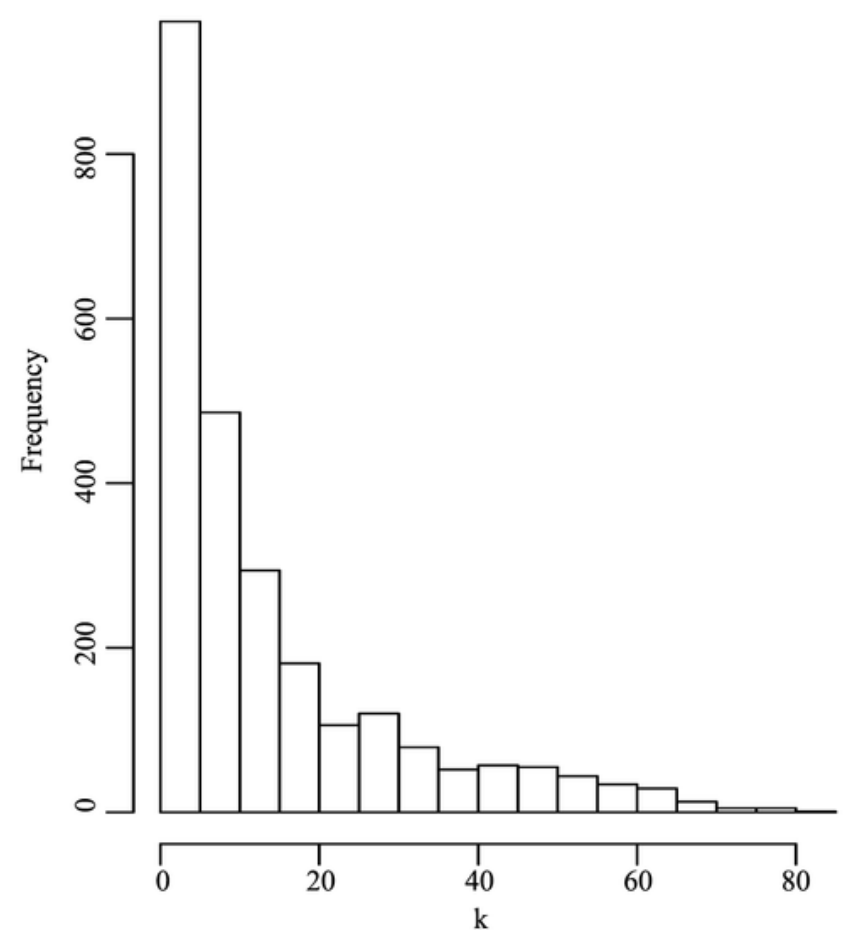

B

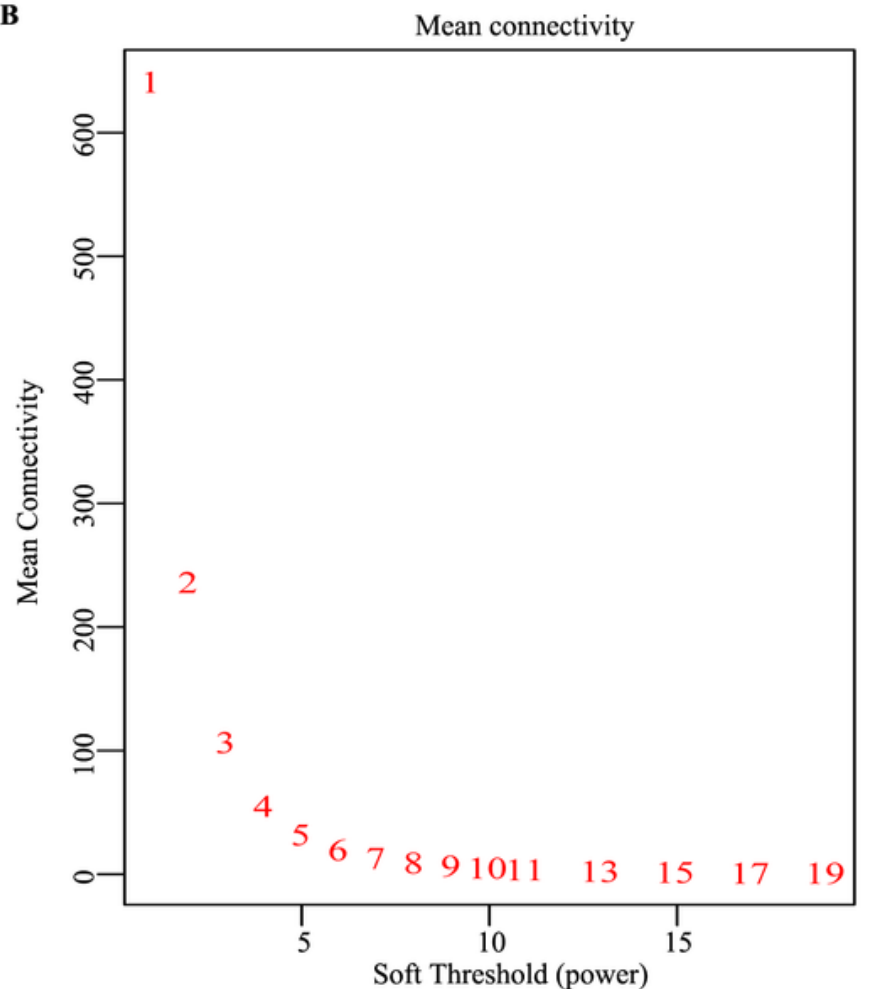

D

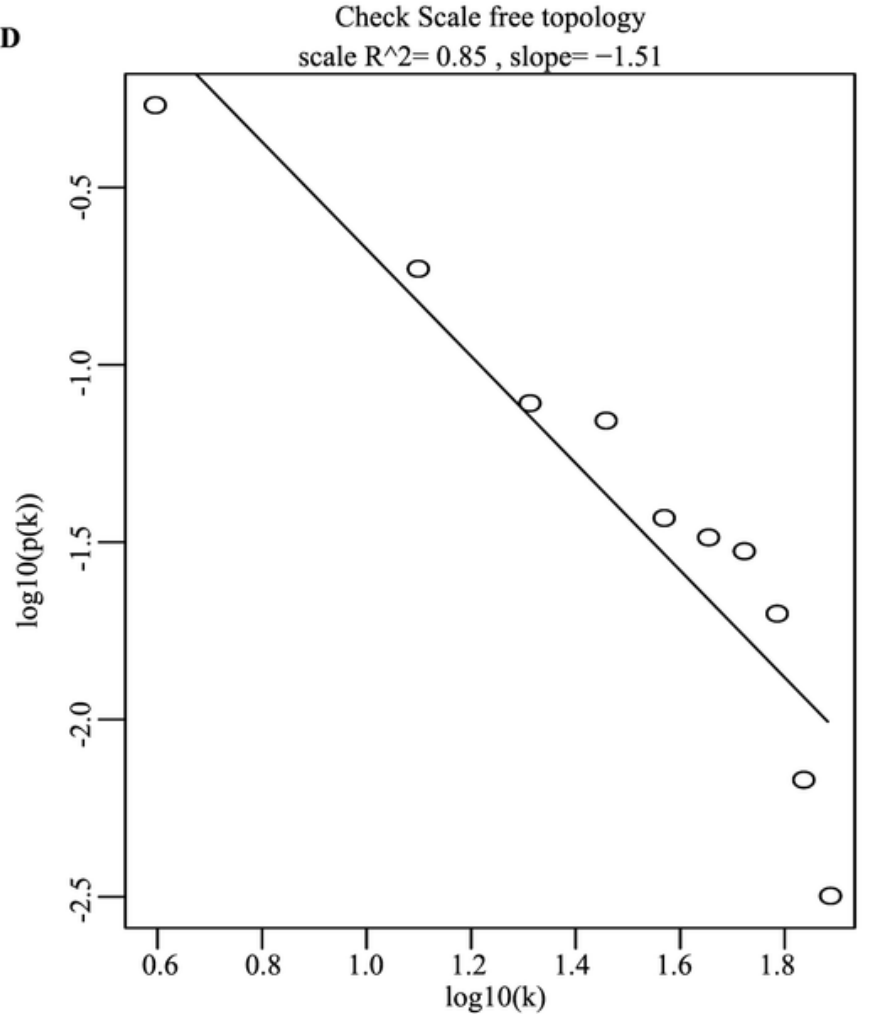

\section{Figure 1}

Determination of soft-thresholding power in the WGCNA. a The scale-free index analysis for softthresholding powers $(\beta)$ in HNSCC. b The mean connectivity analysis for various soft-thresholding powers in HNSCC. $c$ Histogram of connectivity distribution when $\beta=7$ in HNSCC. $d$ Checking the scale-free topology when $\beta=7$ in HNSCC. 
$\mathbf{A}$

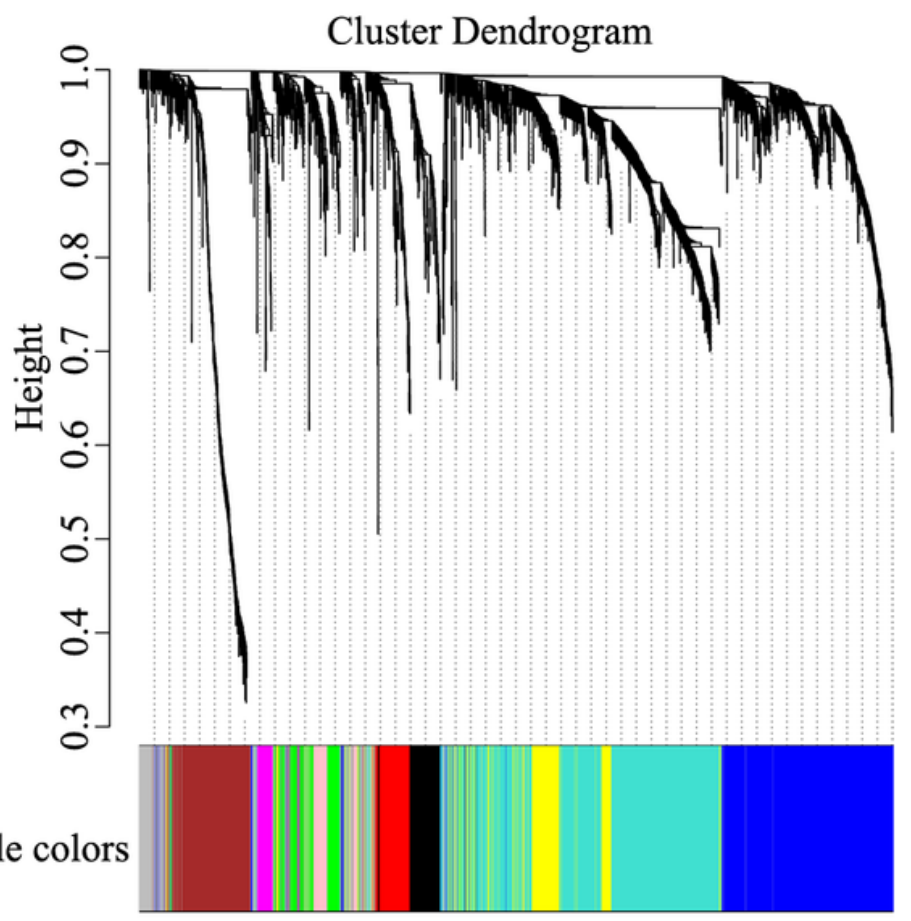

B

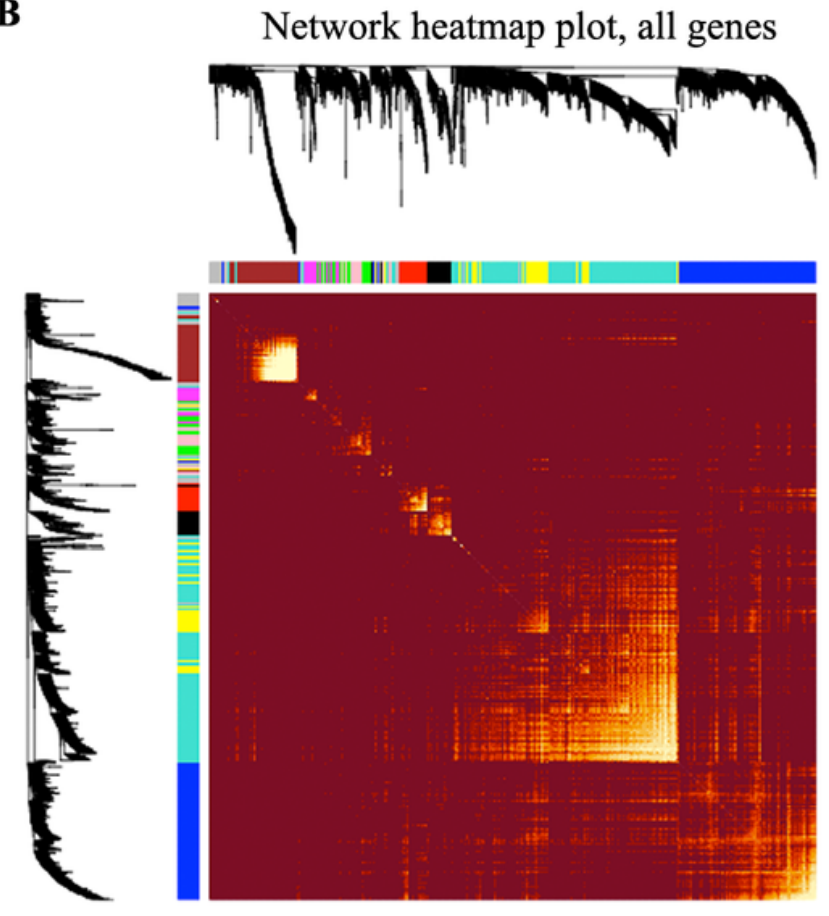

Figure 2

Visualization of WGCNA results. a Hierarchical cluster analysis of genes. Each color represents a module in the constructed gene co-expression network. b The heatmap depicts the Topological Overlap Matrix among all genes in the WGCNA analysis. Light color represents low overlap and progressively darker red color represents higher overlap. 
$\mathbf{A}$

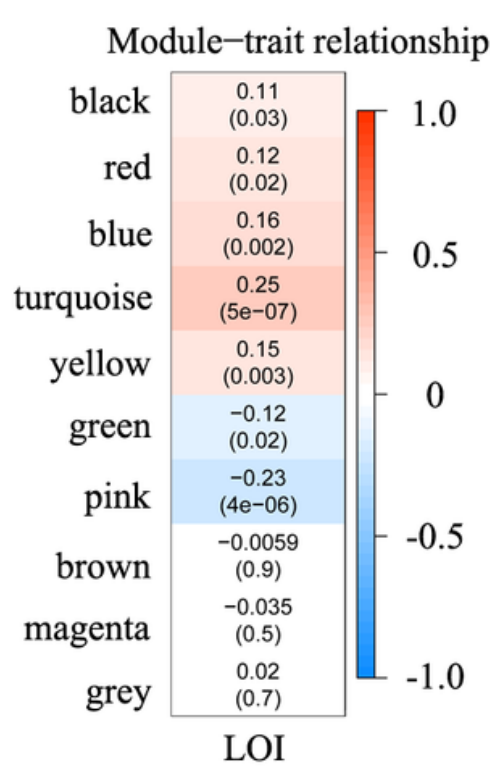

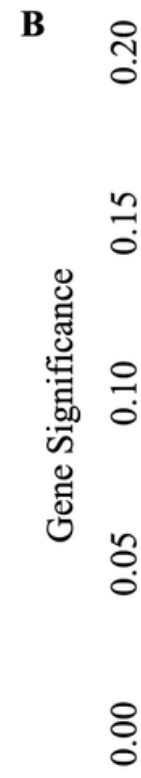

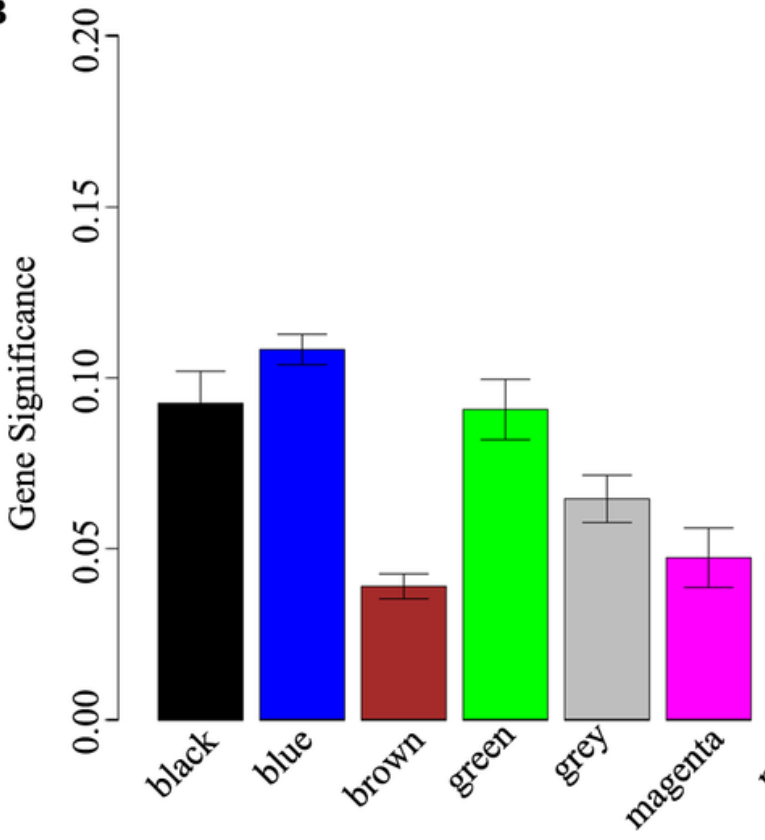

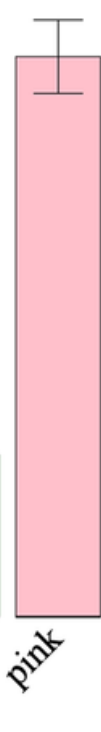

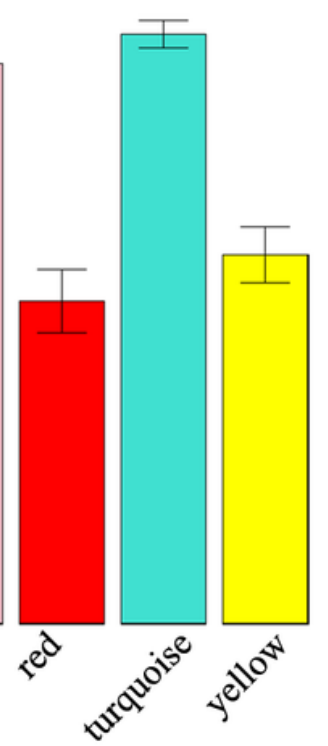

D

C

Module membership vs. gene significance cor $=0.4, \mathrm{p}=1.4 \mathrm{e}-30$

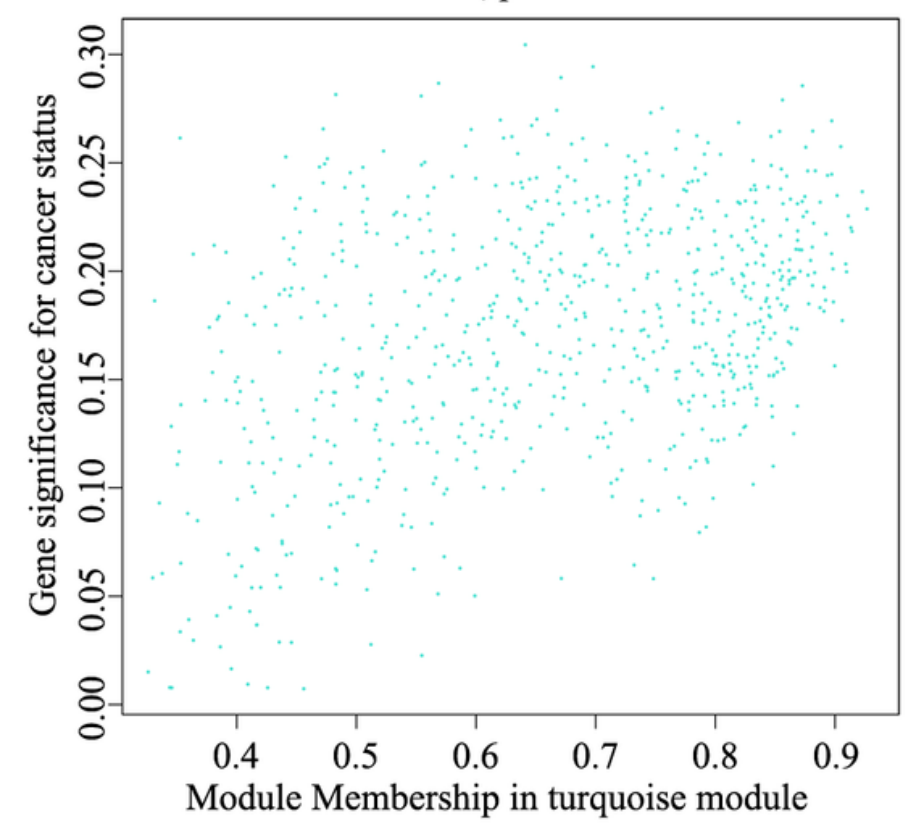

Module membership vs. gene significance cor $=0.59, \mathrm{p}=1.6 \mathrm{e}-08$

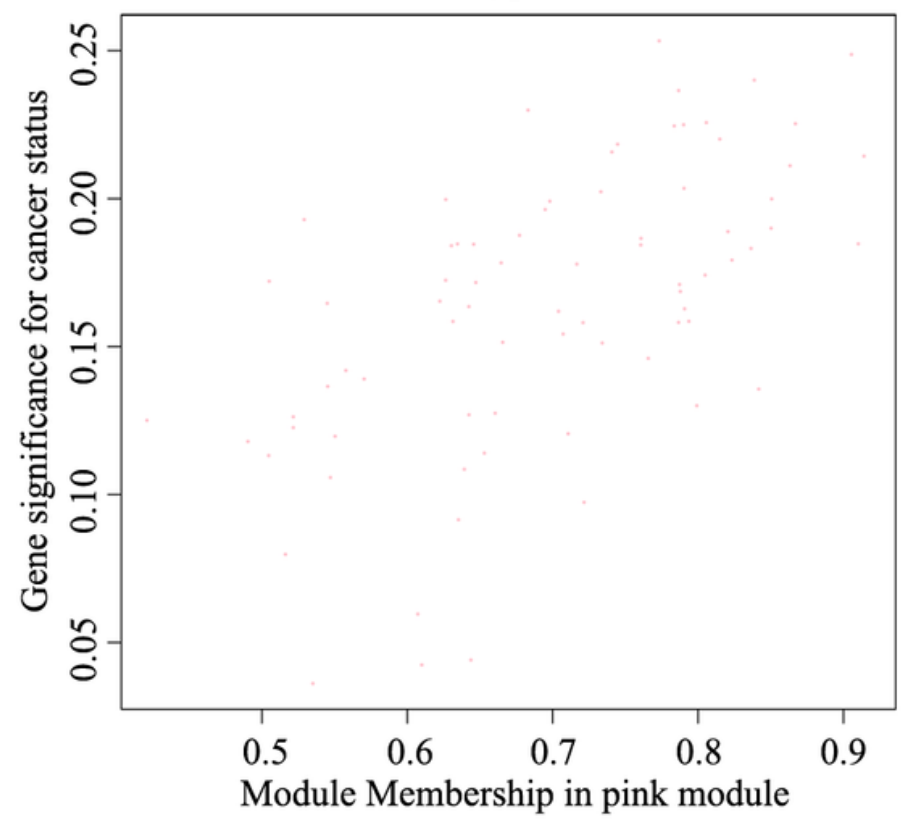

\section{Figure 3}

The correlation analysis of module-trait. a The column corresponds to LOI phenotypic trait, labeled below. Heatmap of each cell at the row-column contains the $p$-value between that module and LOI trait. $b$ Bar plot of the significance level of 10 co-expression modules associated with LOI status. (c and d) Correlation analysis between gene significance of LOI status and module membership in the turquoise (c) and pink (d) modules. 
A

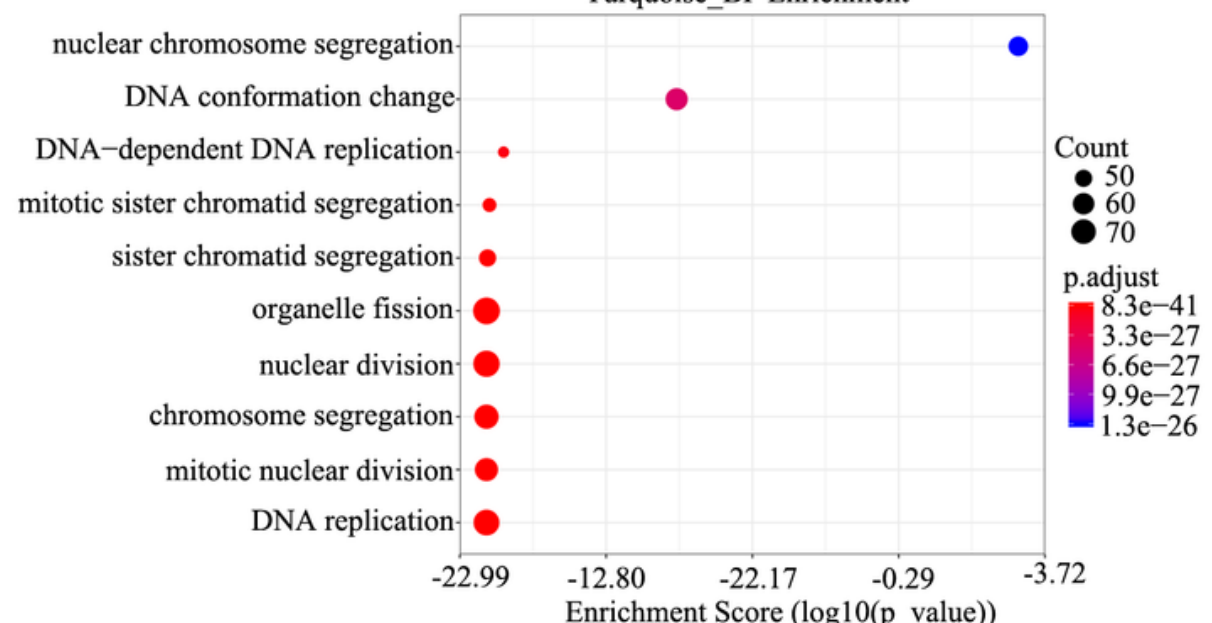

B

Turquoise KEGG Enrichment_GO

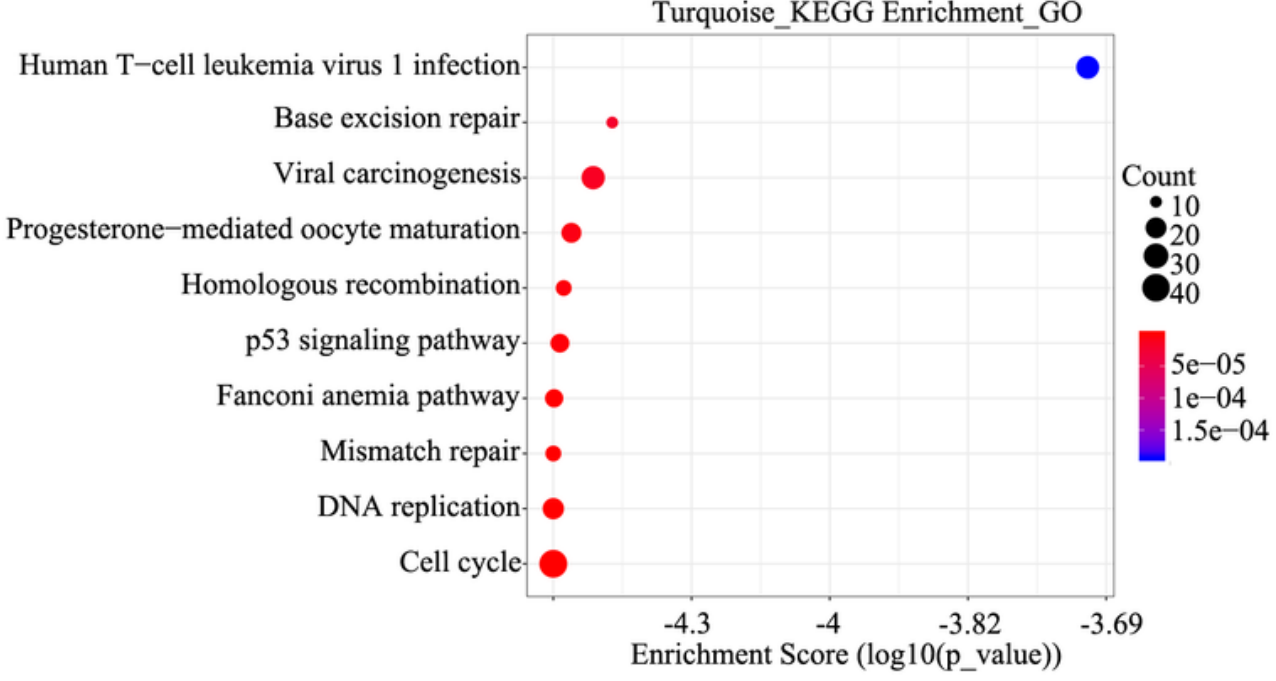

C

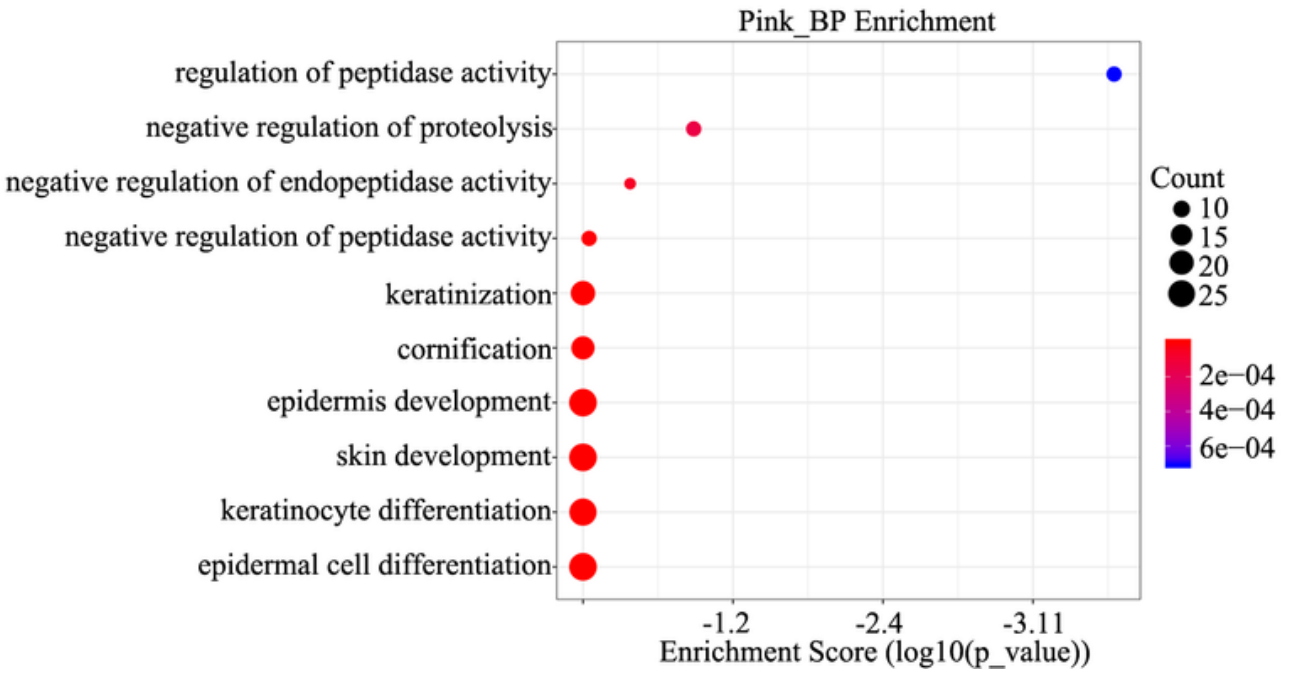

\section{Figure 4}

GO and KEGG analysis in the two key turquoise and pink modules. a Go enriched analysis of turquoise module in the biological process. $b$ Go enriched analysis of turquoise module in the KEGG pathway. c Go enriched analysis of pink module in the biological process. 
$\mathbf{A}$

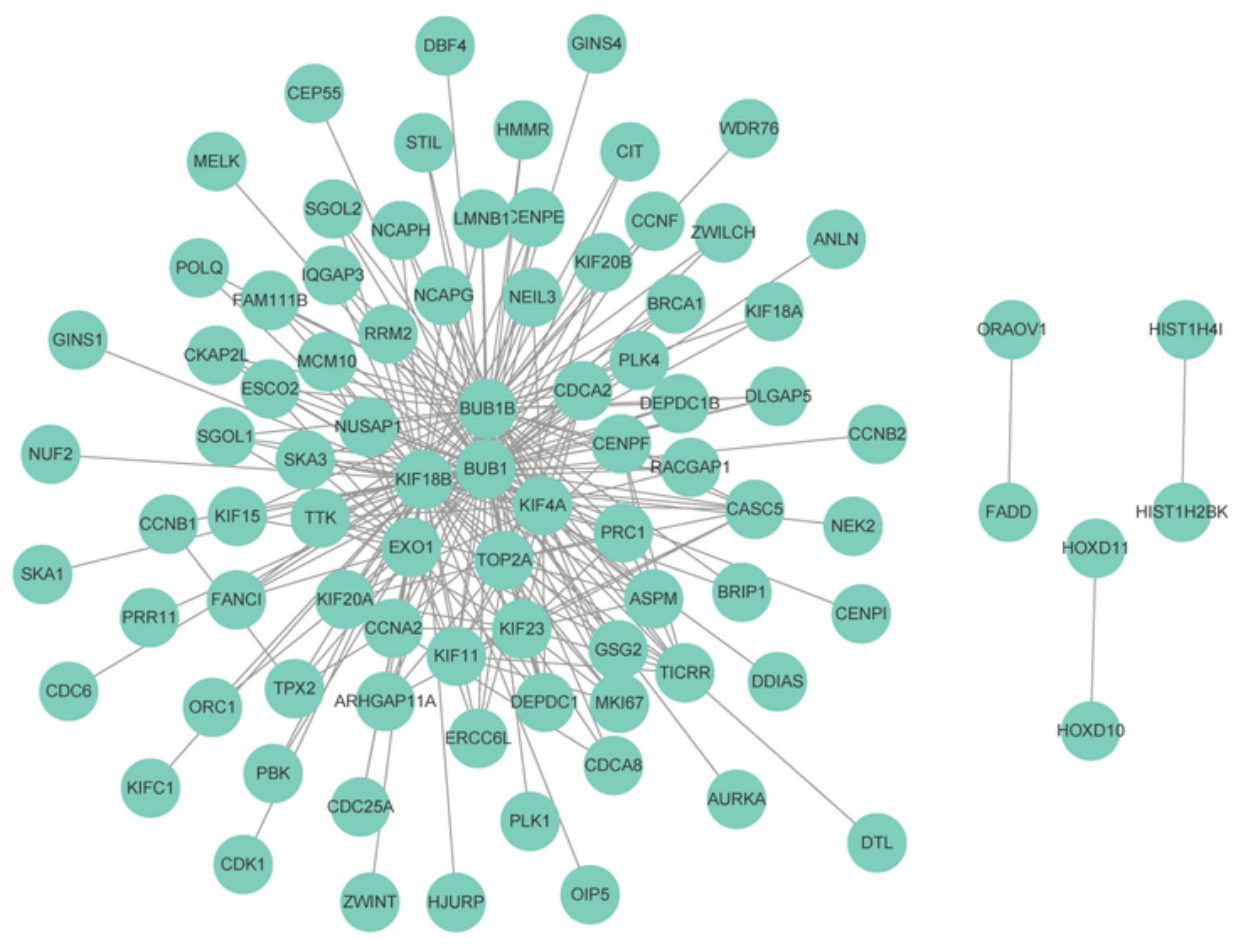

B
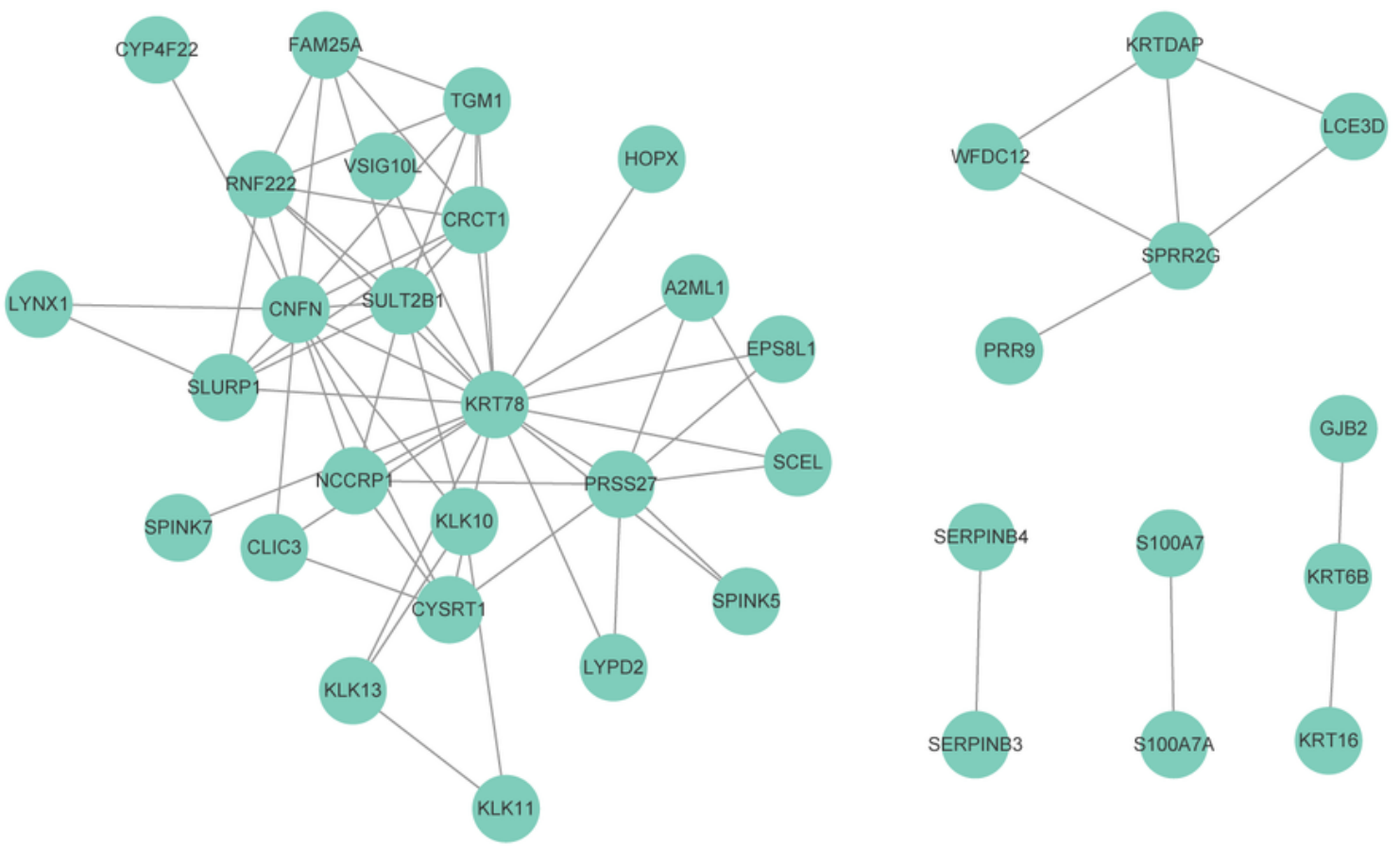

Figure 5

Hub genes identified by PPI network in the modules. ( $a$ and b) PPI interaction network of the DEGs in the turquoise module (a) and pink module (b). 

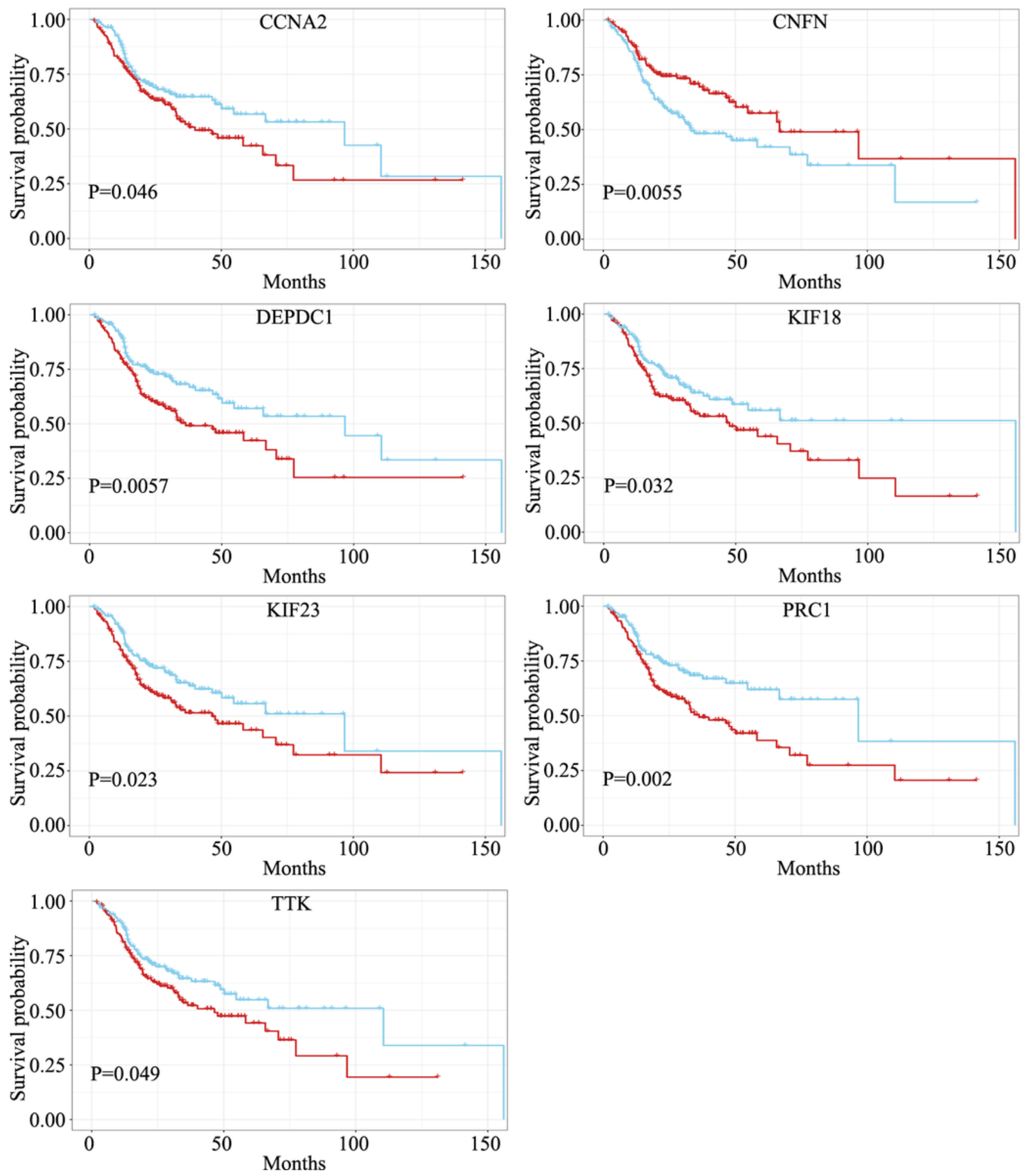

Figure 6

The prognostic value of seven hub genes in HNSCC. Ten years survival analysis of seven hub genes (CCNA2, CNFN, DEPDC1, KIF18, KIF23, PRC1 and TTK1). 
$\mathbf{A}$

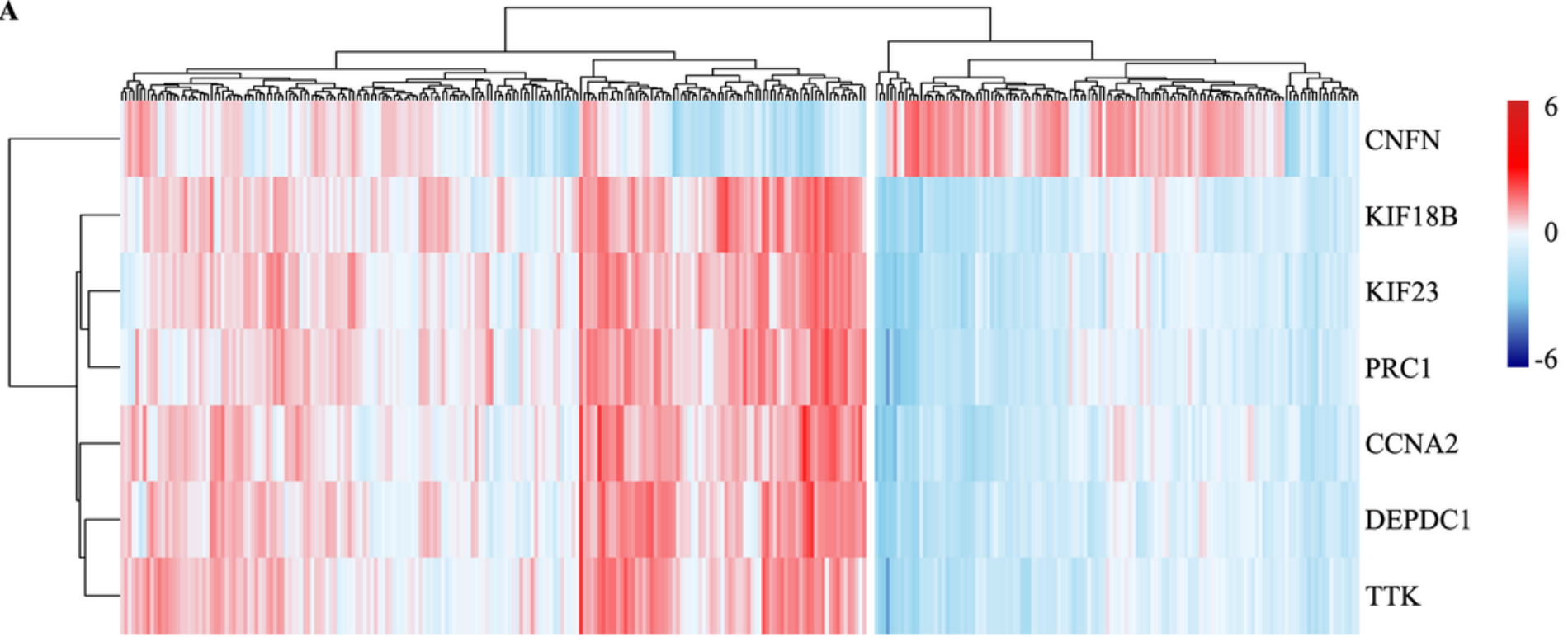

B

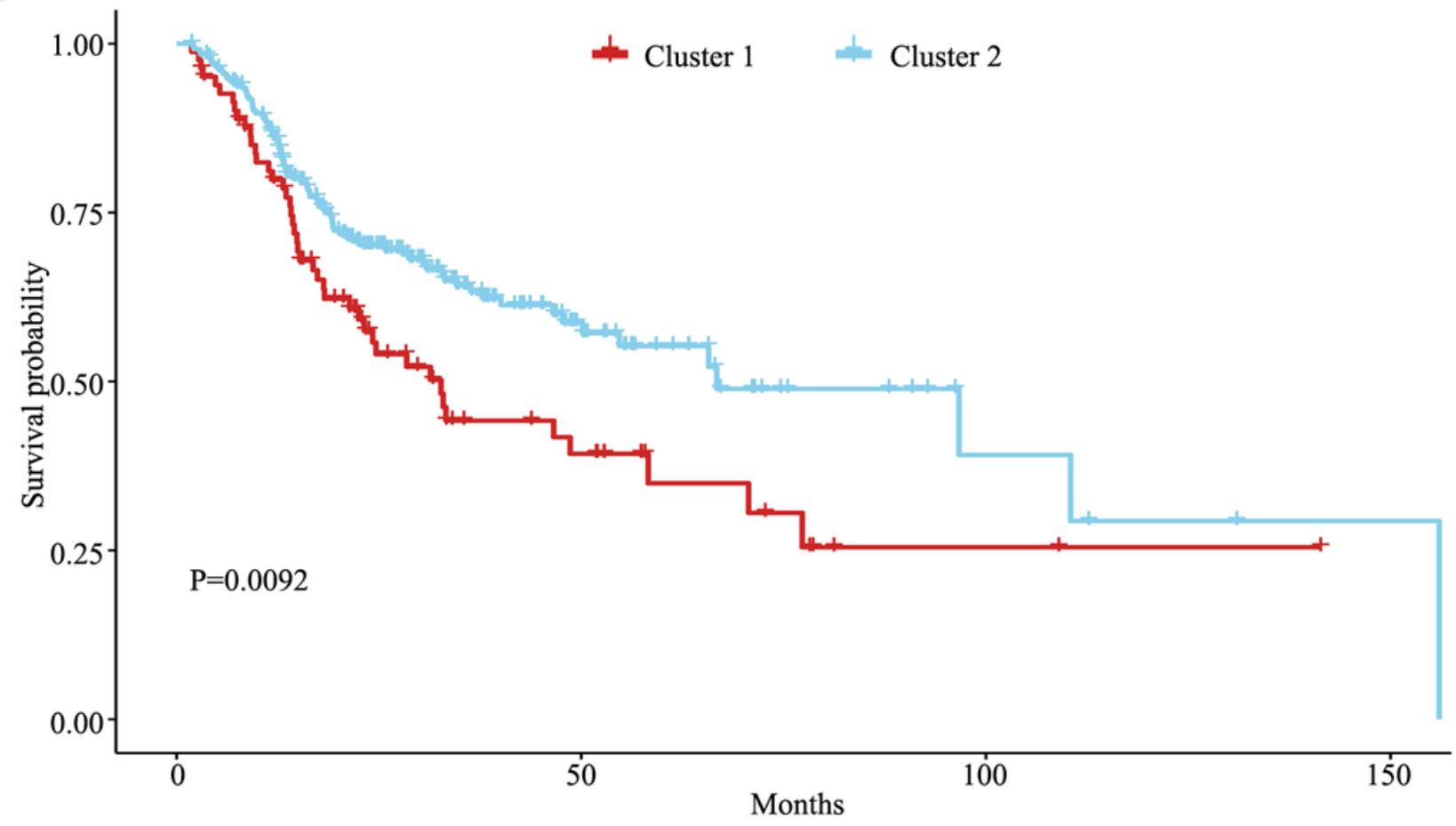

Figure 7

Cluster analysis and cumulative survival of HNSCC patients stratified by hub genes in HNSCC. a Cluster analysis of the seven hub genes (CCNA2, CNFN, DEPDC1, KIF18, KIF23, PRC1 and TTK1) set associated with LOI. b Ten years cumulative survival of HNSCC patients stratified by LOI status. 